\title{
On Certain Characterizations of Barely Transitive Groups
}

\author{
Ahmet Arikan (*) - Nadir Trabelsi (**)
}

\begin{abstract}
In the present work we study perfect barely transitive groups in which a point stabilizer has non-trivial Hirsch-Plotkin radical and give characterizations of these groups. We also prove that a perfect barely transitive group has two non-trivial proper subgroups which intersect trivially.
\end{abstract}

\section{Introduction.}

Let $\mathrm{G}$ be a permutation group on an infinite set $\Omega$. If $\mathrm{G}$ acts transitively on $\Omega$ and every orbit of every proper subgroup of $G$ is finite, then $G$ is called a barely transitive group. This class of groups was considered for the first time by Hartley in connection with the Heineken-Mohamed groups. It is wellknown that $G$ can be represented as a barely transitive group if and only if $G$ has a subgroup $H$ of infinite index such that $\operatorname{Core}_{G} H=\bigcap_{g \in G} H^{g}=\langle 1\rangle$ and $|M: M \cap H|$ is finite for every proper subgroup $M$ of $G$. The subgroup $H$ is called a point stabilizer in $G$.

Barely transitive groups are mostly considered in the locally finite case and it is well-known that these groups are locally nilpotent $p$-groups for a prime $p$. In recent years some work has appeared on the non-locally finite case. Almost all such results can be found in the survey article [12].

In [2] locally graded simple non-periodic barely transitive groups are considered and it is proved that all point stabilizers have trivial HirschPlotkin radical (that is, the maximal locally nilpotent normal subgroup is trivial). In the present paper we give certain characterizations of a perfect

(*) Indirizzo dell'A.: Gazi Üniversitesi, Gazi Eğitim Fakültesi, Matematik Eǧitimi Anabilim Dalı, 06500 Teknikokullar, Ankara, Turkey.

E-mail: arikan@gazi.edu.tr

(**) Indirizzo dell'A.: Department of Mathematics Faculty of Sciences University Ferhat Abbas, Setif 19000, Algeria.

E-mail: nadir_trabelsi@yahoo.fr 
simple barely transitive group with a point stabilizer whose Hirsch-Plokin radical is non-trivial.

Let $\boldsymbol{X}$ be a class of groups. If every proper subgroup of a group $G$ is an $\boldsymbol{X}$-group but $G$ itself is not, then $G$ is called a minimal non- $\boldsymbol{X}$ group.

Our main results are as follows:

THEOREM 1.1. Let $G$ be a simple barely transitive group with a point stabilizer $H$. If the Hirsch-Plotkin radical $F(H)$ of $H$ is non-trivial, then $G$ is a periodic finitely generated minimal non-(locally finite) group. If in addition $H$ is an FC-group, then $G$ is a two-generated minimal non $F C$ group.

COROLlary 1.2. Let $G$ be a perfect barely transitive group with a point stabilizer $H$. If $H$ is soluble, then $G$ is a finitely generated periodic group with every proper subgroup locally finite. If in addition $H$ is an $F C$ group, then $G$ is a two-generated minimal non-FC group.

CoRollary 1.3. Let $G$ be a perfect barely transitive group with a point stabilizer $H$. If $H$ is hypercentral, then either $G$ is locally finite or $G$ is a finitely generated periodic group with every proper subgroup locally finite. If in addition $H$ is an FC-group, then $G$ is a two-generated minimal non-FC group.

\section{Certain Characterizations.}

Throughout the paper we use the term "infinitely generated" in the sense that the group is not finitely generated.

The following lemma is well-known. We just here take care to ensure that we have a proper perfect subgroup in the infinitely generated case.

LEMMA 2.1. If $G$ is a simple infinitely generated group, then $G$ contains a non-trivial proper perfect subgroup.

Proof. We argue as in 1.B.3 Proposition of [9]. Since $G$ is non-abelian, there exist $a, b \in G$ such that $c:=[a, b] \neq 1$. By assumption $\left\langle c^{G}\right\rangle=G$. Hence $G$ has a finitely generated subgroup $Y$ such that $\langle a, b\rangle \leq\left\langle c^{Y}\right\rangle$. Put $Z:=\langle c, Y\rangle$, thus we have $c \in\left\langle c^{Z}\right\rangle^{\prime}$ and hence $\left\langle c^{Z}\right\rangle=\left\langle c^{Z}\right\rangle^{\prime}$. Since $\left\langle c^{Z}\right\rangle \leq Z \neq G$, we have that $\left\langle c^{Z}\right\rangle$ is a proper perfect subgroup of $G$. 
Now we give a characterization of perfect periodic non-locally finite barely transitive groups. We shall also see that the non-existence of perfect subgroups in the group plays a crucial role in determining the finitely generated structure. For example the non-existence of involutions in the group gives finite generation.

THEOREM 2.2. Let $G$ be a non-(locally finite) barely transitive group. Then $G$ has a finitely generated subgroup $F$ such that $\left\langle F^{G}\right\rangle=G$ and $a$ simple barely transitive homomorphic image $\bar{G}$. Furthermore, if $G$ is periodic and infinitely generated, then $\bar{G}$ is infinitely generated and generated by involutions, and is a union of a chain of infinite non-simple perfect subgroups generated by involutions.

Proof. Assume that $G$ has no finitely generated subgroup $F$ such that $\left\langle F^{G}\right\rangle=G$. As in the first paragraph of proof of the Theorem of [2], $G$ is countable and hence we can find a chain of finitely generated subgroups $F_{i}$ of $G$ for $i \in Z^{+}$such that $G=\bigcup_{i=1}^{\infty}\left\langle F_{i}^{G}\right\rangle$. Since every proper normal subgroups of $G$ is locally finite, we see that $G$ is locally finite. But this is a contradiction. So $G$ has a finitely generated subgroup $F$ such that $\left\langle F^{G}\right\rangle=G$.

Let $H$ be a point stabilizer of $G$. As every normal subgroup of $G$ does not contain $F, G$ has a maximal normal subgroup $N$ and $\bar{G}:=G / N$ is a simple barely transitive group with a point stabilizer $\bar{H}$.

Now assume that $G$ is periodic and infinitely generated. We first assume that $G$ is a $p$-group. If $\bar{G}$ is not finitely generated, then $\bar{G}$ contains a proper non-trivial perfect subgroup $\bar{P}$ by Lemma 2.1. Put $\bar{X}=\operatorname{Core}_{\bar{P}}(\bar{H} \cap \bar{P})$, then $\bar{P} / \bar{X}$ is a finite $p$-group by hypothesis. But since $\bar{P}$ is perfect, we have $\bar{P}=\bar{X} \leq \bar{H}$. By arguing similarly, we obtain that $\bar{P} \leq \bar{H}^{\bar{g}}$ for every $\bar{g} \in \bar{G}$ as every $\bar{H}^{\bar{g}}$ is also a point stabilizer in $\bar{G}$. Then

$$
\bar{P} \leq \bigcap_{\bar{g} \in \bar{G}} \bar{H}^{\bar{g}}=\langle 1\rangle .
$$

But this is a contradiction. Hence $\bar{G}$ is finitely generated and thus $G$ has elements $y_{1}, \ldots, y_{s}$ such that $G=\left\langle y_{1}, \ldots, y_{s}\right\rangle N$. Put $F=\left\langle y_{1}, \ldots, y_{s}\right\rangle$ and $E=\operatorname{Core}_{F}(H \cap F)$. Then $F / E(F \cap N)$ is finite, since $F$ is not equal to $G$. Since $G / N$ has no proper subgroup of finite index, we have $F=E(F \cap N)$. Then $G=E(F \cap N) N=H N$. But then $|G: H|=|N: N \cap H|$ is finite, a contradiction. 
So $G$ is not a $p$-group. Now we have that $\bar{G}$ is also infinitely generated by the above argument. Let $\bar{T}$ be the subgroup of $\bar{G}$ generated by all 2-elements in $\bar{G}$. Now $\bar{T}$ is normal in $\bar{G}$ and hence either $\bar{G}=\bar{T}$ or $\bar{T}=\langle 1\rangle$. First assume that $\bar{T}=\langle 1\rangle$ and let $\bar{P}$ be a proper non-trivial perfect subgroup of $\bar{G}$. Then $\bar{P}$ contains a normal subgroup $\bar{X} \leq \bar{H}$ such that $\bar{P} / \bar{X}$ is finite by hypothesis. Since $\bar{G}$ is periodic and has no involution, $|\bar{P} / \bar{X}|$ is odd and hence, by the Feit-Thompson theorem, $\bar{P}=\bar{X} \leq \bar{H}$. Arguing as in the third paragraph we have $\bar{P}=\langle 1\rangle$, a contradiction. Consequently, $\bar{G}=\bar{T}$, i.e. $\bar{G}$ is generated by 2-elements.

Let $\bar{c}$ be an involution in $\bar{G}$; then $\langle\bar{c} \bar{G}\rangle=\bar{G}$ and hence $[\bar{c}, \bar{G}]=\bar{G}$. So $\bar{G}$ is generated by involutions, namely the conjugates of $\bar{c}$ and $\bar{G}$ has elements $\bar{y}_{1}, \ldots, \bar{y}_{r}$ such that $\bar{c}=\left[\bar{c}, \bar{y}_{1}\right] \ldots\left[\bar{c}, \bar{y}_{r}\right]$. Thus $\bar{G}$ has a finitely generated subgroup $\bar{Y}_{1}$ containing $\bar{c}$ such that $\left\langle\bar{c}, \bar{y}_{1}, \ldots, \bar{y}_{r}\right\rangle \leq\langle\bar{c}\rangle \leq\left\langle\bar{c}, \bar{Y}_{1}\right\rangle \neq \bar{G}$. Now we have $\bar{c} \in\left\langle\bar{c}^{\bar{Y}_{1}}\right\rangle^{\prime}$ and hence $\bar{Q}:=\left\langle\bar{c}^{\bar{Y}_{1}}\right\rangle=\left\langle\bar{c}^{\bar{Y}_{1}}\right\rangle^{\prime}$. So $\bar{Q}$ is a proper perfect subgroup of $\bar{G}$ generated by involutions which are conjugates of $\bar{c}$. We also have that for every finitely generated subgroup $\bar{Z}$ of $\bar{G}, \bar{Z}$ is contained in a finitely generated subgroup $\bar{U}$ such that $\langle\bar{c} \bar{U}\rangle$ is perfect. Hence since $\langle\bar{c} \bar{G}\rangle=\bar{G}$, there is a chain of finitely generated subgroups $\bar{Y}_{i}$ of $\bar{G}$ such that $\left\langle\bar{c}^{\bar{Y}_{i}}\right\rangle$ is a perfect subgroup generated by involutions and

$$
\bar{G}=\bigcup_{i \in Z^{+}}\left\langle\bar{c}^{\bar{Y}_{i}}\right\rangle .
$$

Since $G$ is not locally finite and $N$ is locally finite, $\bar{G}$ is non-locally finite and hence only finitely many $\left\langle\bar{c}^{\bar{Y}_{i}}\right\rangle$ can be finite. Also there is a positive integer $m$ such that for every $n>m, \bar{H} \cap\left\langle\bar{c}^{\bar{Y}_{n}}\right\rangle \neq\langle 1\rangle$ and $\left\langle\bar{c}^{\bar{Y}_{n}}\right\rangle \not \leq \bar{H}$. Hence $\left\langle\bar{c}^{\bar{Y}_{n}}\right\rangle$ is non-simple for every $n>m$. So the proof is complete.

This characterization shows that the structure of infinitely generated barely transitive groups is very complicated. But in the rest of the paper we shall see that in many cases the tendency is to be finitely generated.

COROLlaRY 2.3. Let $G$ be a perfect periodic barely transitive group. If $G$ contains no involution, then $G$ is finitely generated.

Proof. Since $G$ is periodic we see that no section of $G$ contains an involution. But if $G$ is infinitely generated, with the notation of Theorem 2.2, $\bar{G}$ is generated by involutions, and we have a contradiction. 


\section{Proof of Theorem 1.1.}

Proof of the Theorem 1.1. - By the Theorem in [2], we have that $G$ must be periodic and $G$ is not locally finite by [8]. Assume that $G$ is not finitely generated. Since $G$ is countable (see [2]), we can write $G=\left\{x_{i}: i \in Z^{+}\right\}$. Define $Y_{n}=\left\{x_{1}, \ldots, x_{n}\right\}, X_{n}=\left\langle H, Y_{n}\right\rangle, R_{n}=$ Core $_{X_{n}} H$ as in [2] and [6]. As in the proof of Lemma 2.1 of [2], $H$ is not contained in maximal subgroup of $G$ and hence $X_{n} \neq G$ for every positive integer $n$. We have $G=\bigcup_{i=1}^{\infty} Y_{i}, R_{n} \unlhd X_{n}$ and $\left|X_{n} / R_{n}\right|$ is finite.

We first show that $F\left(R_{n}\right) \neq\langle 1\rangle$ for all $n \geq 1$. For the contrary assume that $F\left(R_{n}\right)=\langle 1\rangle$ for some $n \in Z^{+}$. Put $P=F(H)$, then $P R_{n} / R_{n} \simeq P$ is finite. Hence $H$ has a non-trivial finite normal subgroup. This implies that $\left|H: C_{H}(P)\right|$ and thus $\left|H: C_{H}(g)\right|$ are both finite for every $g \in P$. This implies that $F C_{G}(H) \neq\langle 1\rangle$, since $H$ is inert in $G$ (see [4] for the definitions). Now by 2.2 of [4], we have $F C_{G}(H)=G$. By the proof of Proposition 2 of [12], $G$ is now a minimal non- $F C$ group. It is well-known that $G$ is either a locally nilpotent $p$-group or $G$ is quasi-finite by Theorem 1 of [3] and [10]. If $G$ is locally nilpotent $p$-group, then it is not simple, a contradiction. If $G$ is quasi-finite, then $G$ is two-generated by [14] p. 97 . But this is again a contradiction. Now we have that $F\left(R_{n}\right) \neq\langle 1\rangle$ and $P$ is infinite. Clearly we also have that $F\left(X_{n}\right) \neq\langle 1\rangle$. Let $E$ be a finitely generated subgroup of $G$, then arguing as in the final paragraph of the proof of Lemma 3 of [6], we have $E$ is nilpotent-by-finite, in particular $Y_{n}$ is nilpotent-by-finite for every positive integer $n$. Now by [4], $H / P$ is an $F C$-group and then $H / T$ is locally finite, where $T / P=Z(G / P)$. Thus $T$ is (locally nilpotent)-by-abelian. Clearly $H \cap Y_{n}$ has finite index in $Y_{n}$ and thus it is finitely generated. Since $\left(H \cap Y_{n}\right) T / T \cong H \cap Y_{n} / H \cap Y_{n} \cap T$ is finite, $H \cap Y_{n} / T \cap Y_{n}$ is finite. Put $T_{n}=T \cap Y_{n}$, then $\left|Y_{n}: T_{n}\right|=\left|Y_{n}: H \cap Y_{n}\right|\left|H \cap Y_{n}: T_{n}\right|$ is finite. This implies that $T_{n}$ is finitely generated and thus $T_{n}$ is nilpotent-byabelian. As $Y_{n}$ is nilpotent-by-finite, it has a normal nilpotent subgroup $K_{n}$ such that $Y_{n} / K_{n}$ is finite. Now $K_{n}$ is a finitely generated nilpotent subgroup of $Y_{n}$ and clearly it is finite. Thus $Y_{n}$ is finite for every positive integer $n$. Since $G$ is the union of $Y_{i}$ for $i \geq 0, G$ is locally finite. But this is a contradiction.

Now we may assume that $G$ is finitely generated. If $H$ is not an $F C$ group, then by [4] Theorem 1.4, $H / P$ is an $F C$-group. Hence $H^{\prime} / H^{\prime} \cap P$ is locally finite by [14] Theorem 4.32. Now by [4] Theorem 1.4 (ii), $P$ is a $p$ group for some prime $p$ and hence it is locally finite. This implies that $H^{\prime}$ is locally finite. Since $H$ is locally finite-by abelian, it is locally finite. As 
$|K: K \cap H|$ finite for every proper subgroup $K$ of $G, K$ is locally finite, i. e. every proper subgroup of $G$ is locally finite. Consequently, $G$ is a minimal non-(locally finite) group.

If $H$ is an $F C$-group, then as above $G$ is a minimal non- $F C$ group and hence $G$ is two-generated by [3] Theorem 1 and above similar arguments.

Proof of Corollary 1.2. - If $G$ is locally finite, then by Theorem 1 of [5] $G$ is a $p$-group and Theorem 2 of [5] (or [1]) $G$ cannot be perfect, a contradiction. We also have that $G$ has a maximal proper normal subgroup $N$ such that $\bar{G}=G / N$ is a simple barely transitive group with a point stabilizer $\bar{H}$ by Theorem 2.2. Since $\bar{H}$ is non-trivial soluble, we have $F(\bar{H}) \neq\langle 1\rangle$ and hence by Theorem $1.1, \bar{G}$ is finitely generated and periodic with all proper subgroups locally finite. Let $K$ be a proper subgroup of $G$ and $E$ be a finitely generated subgroup of $K$. Since $E N \neq G$, we have that $E / N \cap E$ is finite and hence $N \cap E$ is finitely generated. As $N$ is locally finite, $N \cap E$ is finite and thus $E$ is finite. This means that $K$ is locally finite, i.e. every proper subgroup of $G$ is locally finite. The other properties follow as in the proof of Theorem 1.2.

The proof of Corollary 1.3 is also similar. But it is not known yet whether a locally nilpotent barely transitive $p$-group with all proper subgroups hypercentral is not perfect.

COROLlaRY 3.1. Let $G$ be a simple barely transitive group with a point stabilizer $H$. If $C_{G}(H) \neq\langle 1\rangle$, then $G$ is a periodic finitely generated minimal non-(locally finite) group.

Proof. Let $1 \neq a \in C_{G}(H)$, then $W:=H\langle a\rangle \neq G$. Since $G$ is simple, we have that $W$ is a point stabilizer in $G$ with $a \in F(W)$. Hence the result follows by Theorem 1.1.

\section{Intersections of subgroups.}

In this section we consider trivial intersections of proper subgroups of barely transitive groups. The classification in [15] is very useful for barely transitive groups, since every proper subgroup is residually finite.

THEOREM 4.1. Let $G$ be a perfect barely transitive group, then $G$ has two proper non-trivial subgroups which intersect trivially. 
Proof. Assume that the assertion is false. First suppose that $G$ is periodic. Then since every proper subgroup of $G$ is residually finite, by [15] every proper subgroup of $G$ is isomorphic either to a finite group of (generalized) quaternions or to a finite cyclic $p$-subgroup. Then clearly every proper subgroup of $G$ is finite.

If $G$ contains a quaternion group, then since a quaternion group is a 2 group and proper subgroups of $G$ intersect non-trivially, $G$ is a 2-group. It is well-known that every infinite 2-group contains an infinite abelian subgroup, a contradiction. So $G$ cannot contain a quaternion group. Since $G$ is perfect, $G$ must be two-generated. In this case $G$ has a subgroup $E$ of order $p$ for a prime $p$ which is contained in every proper subgroup of $G$. Clearly $E$ is a normal subgroup of $G$, so for every proper subgroup $H$ of $G$, Core $_{G} H \neq\langle 1\rangle$. But this is a contradiction.

Now assume that $G$ is non-periodic. By hypothesis $G$ must be torsionfree. Let $K$ be a proper subgroup of $G$, then $K$ is residually finite by [2] p. 3 and hence $K$ is isomorphic to a proper subgroup of $(Q,+)$ the additive group of rational numbers by [15]. So every proper subgroup of $G$ is abelian. This implies that $G$ is two-generated, say $G=\langle a, b\rangle$. Then $K:=\langle a\rangle \cap\langle b\rangle \neq\langle 1\rangle$ by hypothesis. Hence $K$ is a proper normal subgroup of $G$. But this gives a contradiction, since $G$ is simple-see [13], p. 85.

Acknowledgments. Both authors are grateful to the referee for the careful reading and many useful comments.

\section{REFERENCES}

[1] A. ARIKAN, On barely transitive p groups with soluble point stabilizer, J. Group Theory, 5, No. 4 (2002), pp. 441-442.

[2] A. ARIKAN, On locally graded non-periodic barely transitive groups, Rend. Semin. Mat. Univ. Padova, 117 (2007), pp. 141-146.

[3] V. V. Belyaev, Groups of the Miller-Moreno type, (Russian) Sibirsk. Mat. Z., 19, no. 3 (1978), pp. 509-514.

[4] V. V. BELYAEV, Inert subgroups in infinite simple groups (Russian). Sibirsk. Math. Zh., 34 (1993), pp. 17-23; translation in Siberian Math. J., 34 (1993), pp. 606-611.

[5] V. V. Belyaev - M. KuzucuoĞLu, Locally finite barely transitive groups (Russian), Algebra i Logika, 42 (2003), pp. 261-270; translation in Algebra and Logic, 42 (2003), pp. 147-152.

[6] M. R. Dixon - M. J. Evans - H. SMith, Groups with all proper subgroups soluble-by-finite rank, J. Algebra, 289 (2005), pp. 135-147.

[7] B. HARTLEY, On the normalizer condition and barely transitive permutation groups, Algebra and Logic, 13 (1974), pp. 334-340. 
[8] B. Hartley - M. KuzucuoĞLu, Non-simplicity of locally finite barely transitive groups, Proc. Edin. Math. Soc., II. Ser., 40, No. 3 (1997), pp. 483-490.

[9] O. H. Kegel - B. A. F. Wehrfritz, Locally Finite Groups, North-Holland Math. Library, vol. 3, North-Holland, Amsterdam, 1973.

[10] M. KuzucuoĞLu - R. E. Phillips, Locally finite minimal non-FC-groups. Math. Proc. Cambridge Philos. Soc., 105, no. 3 (1989), pp. 417-420.

[11] M. KuzucuoĞLu, Barely transitive permutation groups, Arch. Math., 55 (1990), pp. 521-530.

[12] M. KUZUCUoĞLU, On torsion free barely transitive groups, Turkish J. Math., 24 (2000), pp. 273-276.

[13] M. KuZucuoĞLu, Barely transitive groups, Turkish J. Math., 31 (2007), Suppl. pp. 79-94.

[14] D. J. S. Robinson, Finiteness Conditions and Generalized Soluble Groups, Vols. 1 and 2, ( Springer -Verlag 1972).

[15] A. I. SozUTov, Residually finite groups with nontrivial intersections of pairs of subgroups, Sibirsk. Mat. Zh., 41, no. 2 (2000), pp. 437-441, iv; translation in Siberian Math. J., 41, no. 2 (2000), pp. 362-365.

Manoscritto pervenuto in redazione il 6 maggio 2009. 